\title{
A Teoria da Credibilidade da Política Monetária
}

\author{
The Theory of Monetary Policy Credibility
}

HELDER FERREIRA DE MENDONÇA*

\begin{abstract}
RESUMO: Este artigo analisa os principais argumentos relativos à teoria da credibilidade da autoridade monetária da literatura corrente. Nesse sentido, a origem do debate intitulado regras versus discrição é examinada e os avanços na literatura da proposta de independência do banco central. Os resultados indicam que o problema do viés inflacionário para a condução da política monetária é resultado de um conjunto de hipóteses ad hoc usadas pela literatura. Assim, os desenvolvimentos na literatura sobre a credibilidade do viés inflacionário representam um caso particular para a teoria monetária.

PALAVRAS-CHAVE: Política monetária; credibilidade; regras versus discrição.
\end{abstract}

ABSTRACT: This paper analyzes the main arguments concerning credibility theory for monetary authority from the mainstream literature. In this sense, the origin of the debate entitled rules versus discretion is examined and the advances in literature from the central bank independence proposal. The findings denote that the problem of the inflationary bias for conduction of monetary policy is a result of a set of ad hoc hypotheses used by the literature. Thus, the developments in the literature concerning the credibility from inflationary bias represent a particular case for monetary theory.

KEYWORDS: Monetary policy; credibility; rules versus discretion.

JEL Classification: E52; E58.

\section{INTRODUÇÃO}

Nos últimos trinta anos, a discussão sobre quais objetivos a política monetária deve perseguir e como deve ser conduzida, tem recebido a atenção de diversos economistas. Apesar das divergências teóricas, até o princípio dos anos 70 a política monetária era entendida como um instrumento de política econômica que deveria ser utilizado tendo como principal objetivo a sustentação de elevados níveis de emprego. Em meados dos anos 80, porém, o principal objetivo da política monetária tornou-se a busca da estabilidade dos preços. * Professor do Departamento de Economia da Universidade Federal Fluminense - UFF, Rio de Janeiro/
RJ, Brasil. E-mail: helderfm@hotmail.com; Orcid: 0000-0003-1465-1755. 
A mudança na análise teórica e na prática da política monetária teve como principal marco o período compreendido entre 1971 e 1973 . No aspecto prático, o fim do sistema de Bretton Woods simultâneo ao aumento dos preços em escala internacional proveniente do primeiro grande choque do petróleo, implicou a necessidade do encontro de uma nova âncora nominal capaz de controlar o nível de preços na economia. A estratégia utilizada por diversos países consistiu no uso de metas para o crescimento dos agregados monetários, o que por sua vez denotava uma estrutura para a condução da política monetária baseada em regras. ${ }^{1}$ Assim, nessa fase de inflexão, pode-se dizer que a possível influência teórica sobre os responsáveis pelapolítica monetária deve ser atribuída à Friedman² e não à propalada revolução das expectativas racionais.

Por outro lado, levando-se em conta o mesmo período supracitado (19711973) a análise teórica da política monetária sofreu mudanças significativas a partir da introdução do conceito de expectativas racionais ${ }^{3}$ à teoria econômica. ${ }^{4}$ Este elemento foi de crucial importância porque implicou uma transformação na interpretação do comportamento dos agentes frente à política monetária. De acordo com a interpretação desenvolvida por Lucas e Sargent, que ficou conhecida como novo-clássica, os agentes fazem uso da melhor forma possível das informações disponíveis. Logo, na ausência de barreiras informacionais, a possibilidade de ilusão monetária, elemento-chave para explicar a existência do trade-off na curva de Phillips (versão Friedman-Phelps) deixa de existir. Em outras palavras, variações sistemáticas na política monetária, sob expectativas racionais, não são capazes de afetar o lado real da economia.

Sob a égide do conceito de expectativas racionais surgem os economistas neokeynesianos nos anos 80 . O argumento central desses teóricos consiste no reconhecimento da idéia keynesiana de que a economia não se ajusta de forma instantânea e suave a choques (inclusive monetários) sendo necessária a incorporação dos fundamentos microeconômicos. Em outras palavras, a principal característica desses teóricos é o uso de um modelo dinâmico de equilíbrio geral, combinado a expectativas racionais, com rigidez temporária dos preços nominais.

Concomitante às transformações que a teoria macroeconômica vinha sofrendo, uma nova discussão teve início a partir da questão de como minimizar perdas sociais quando medidas políticas precisam ser tomadas em diversos períodos no

\footnotetext{
${ }^{1}$ Exemplos de grandes economias que fizeram uso da estratégia de metas monetárias foram Alemanha, Japão e EUA.

${ }^{2}$ Friedman (1968), propôs a adoção de uma regra para a expansão dos agregados monetários como forma de evitar flutuações significativas sobre a taxa de inflação.

${ }^{3}$ A hipótese de expectativas racionais teve origem com Muth (1961); seu principal objetivo era procurar conciliar a hipótese da racionalidade, amplamente utilizada na modelagem do comportamento dos agentes econômicos, com o mecanismo de formação de expectativas.

${ }^{4}$ Durante o período sobredito diversos trabalhos de Lucas (1972 e 1973) e Sargent (1973) influenciaram sobremaneira a análise acadêmica.
} 
tempo. A partir de então, um problema potencial que passou a despertar grande interesse na academia consiste em verificar se as autoridades políticas devem agir segundo regras que determinam as ações a serem feitas em qualquer momento, ou se devem otimizar a escolha dos instrumentos políticos à cada período no tempo. Este tema deu origem ao propalado debate regras versus discricionariedade.

Ainda nos anos 70, Kydland \& Prescott (1977) deram início ao estudo sobre a credibilidade $^{5}$ da política monetária ressaltando a análise sobre inconsistência temporal. Na perspectiva destes autores, a utilização de regras para a condução da política monetária representa a melhor solução para que a política atual seja consistente com a política de equilíbrio futura. Seguindo a mesma linha de argumentação, Barro \& Gordon (1983), retomaram a análise efetuada por Kydland \& Prescott e salientaram a importância do papel da reputação sobre a política monetária como forma de disciplinar a condução da política monetária. O ponto central da análise é que a persistência da inflação é atribuída à perda de reputação do governo, devido ao não cumprimento dos acordos previamente firmados com a sociedade. ${ }^{6}$ Devese chamar a atenção para uma variável que, a partir de então, tornou-se comum nos estudos subseqüentes - a existência de estímulos para que a autoridade política inflacione o sistema, isto é, a presença de um viés inflacionário. ${ }^{7}$

A análise realizada pelos autores sobreditos influenciou sobremaneira a literatura que envolve o tripé inflação - banco central — governo. Rogoff (1985) na tentativa de encontrar uma solução para eliminar o viés inflacionário do responsável pela política monetária, propôs a adoção de um banco central independente (BCI) com um presidente conservador (aversão à inflação maior do que a da sociedade). Com base nesta análise, diversos estudos foram elaborados com a necessidade de satisfazer duas condições no equilíbrio (Barro, 1986):

i) é válido o preceito das expectativas racionais, isto é, as expectativas inflacionárias dos agentes são corretas na média; e

ii) embora o responsável pela política tenha o poder em cada período de enganar o público via surpresa inflacionária, ele não é motivado a fazer uso deste mecanismo. Em outras palavras, no equilíbrio, o benefício marginal de uma inflação surpresa deve ser igual ao custo marginal da inflação.

\footnotetext{
${ }^{5} \mathrm{O}$ conceito credibilidade pode ser entendido como o nível de confiança que os agentes econômicos depositam na exeqüibilidade de uma política anunciada. Ou seja, uma política inspirará maior credibilidade se ela sinalizar aos agentes uma chance reduzida da ocorrência de inconsistência temporal. Assim, por exemplo, se o $\mathrm{BC}$ ao longo de sua história obteve êxito no combate à inflação (o que implica conquista de reputação), os agentes acreditam que o BC terá sucesso no controle da inflação futura, o que, por sua vez, denota alto grau de credibilidade.

${ }^{6}$ Um outro importante trabalho para a literatura sobre a conquista de reputação da autoridade monetária, representado por um jogo estratégico entre a autoridade monetária e um sindicato, é apresentado por Backus \& Driffill (1985).

${ }^{7} \mathrm{O}$ conceito de viés inflacionário deriva do argumento da ineficácia das políticas. O âmago do conceito pode ser entendido como a tentação que os governos sofrem de buscar um aumento do produto e/ou redução do nível de desemprego por meio do uso de políticas monetárias expansionistas.
} 
Dentre a vasta literatura sobre o tema, merecem atenção especial as análises desenvolvidas por Walsh (1995) e Svensson (1997). Walsh (1995) adota a estrutura do agente-principal para determinar como a elaboração de contratos para o banco central (BC) deveriam ser estruturados para induzi-lo a adotar a política socialmente ótima. Por outro lado, Svensson (1997) elabora um modelo que mistura o conservadorismo do BC, a utilização de contratos ótimos e metas para a inflação.

Fazendo-se uso dos argumentos apresentados por Clarida, Gali \& Gertler (1999), a literatura sobre credibilidade pode ser dividida em duas vertentes:

i) teórica - é analisado o problema de persistência inflacionária sob o comportamento discricionário da autoridade monetária $;^{8} \mathrm{e}$

ii) aplicação de políticas - se a política monetária não estiver voltada para ocombate à inflação, o processo de desinflação da economia pode implicar um sacrifício social maior que o necessário. ${ }^{9}$

Não obstante a fonte do problema sob o ponto de vista teórico e aplicado serem diferentes, ambas as visões sugerem que o ganho de credibilidade pelo BC pode ser capaz de reduzir a inflação a um custo menor. Nesse sentido, ao longo da década de 80 vários $\mathrm{BCs}$ assumiram a postura de que é necessária a independência do BC (IBC) em relação ao governo para que o viés inflacionário seja eliminado. No período recente, um número expressivo de países passaram a fazer uso da estratégia de metas para a inflação com o objetivo de desinflacionar a economia e/ ou assegurar a estabilidade de preços. ${ }^{10}$

O objetivo do artigo consiste em analisar os principais argumentos sobre a teoria da credibilidade da autoridade monetária a partir da literatura padrão. $\mathrm{O}$ estudo apresenta-se estruturado da seguinte forma: a segunda seção analisa a origem da teoria sobre a credibilidade da autoridade monetária; a terceira organiza a evolução da literatura sobre credibilidade a partir da proposição de IBC; a quarta apresenta a regra de acompanhamento elaborada por Taylor (1993) para a determinação da taxa de juros; por último são apresentadas algumas ponderações aos fundamentos teóricos que sustentam a análise sobre a credibilidade da política monetária.

\footnotetext{
${ }^{8} \mathrm{O}$ principal exemplo de custo associado ao comportamento discricionário é a manifestação do viés inflacionário.

${ }^{9}$ Neste caso, a fonte do problema advém do fato de que salários e preços hoje dependem do que os agentes esperam do comportamento futuro dos preços, o que por sua vez depende do curso da política monetária.

${ }^{10} \mathrm{O}$ primeiro país a adotar o regime de metas inflacionárias explícitas nos anos 90 foi a Nova Zelândia (1990), sendo seguido posteriormente por Canadá (1991), Reino Unido (1992), Suécia (1993), Finlândia (1993), Austrália (1994) e Espanha (1994). Também são exemplos Israel, Chile e Brasil. Além dos países citados há o caso daqueles que utilizam metas inflacionárias implícitas. Esta é situação de: Áustria, Bélgica, Dinamarca, França, Alemanha, Irlanda, Itália, Holanda, Portugal, Japão, Coréia, México, Suíça e EUA.
} 


\section{A GÊNESE DA TEORIA SOBRE CREDIBILIDADE}

Phillips (1957) utilizou uma estrutura com base em equações diferenciais para avaliar a efetividade de políticas de estabilização. Esta análise teve o mérito de mostrar que os resultados das políticas dependem de três elementos básicos: a dinâmica do sistema, o tipo de política adotada e as defasagens no ajuste da política. Destarte, três tipos de políticas discricionárias podem ser identificadas (Argy, 1988):

i) proporcional - os governos reagem à defasagem entre o produto atual e o de pleno emprego;

ii) derivativa - os governos reagem de forma precedente a mudanças no produto; e

iii) integral — os governos reagem à soma dos desvios passados em relação ao nível de pleno emprego.

Além do comportamento discricionário, a conduta da autoridade política na busca dos objetivos pretendidos também pode ser resultado de um comportamento baseado em regras. ${ }^{11}$ A partir dos anos 60 e início dos anos 70 a análise de Friedman (1968) contra o comportamento discricionário das autoridades monetárias recebeu grande atenção. Na interpretação friedmaniana os agentes são dotados de expectativas adaptativas, ${ }^{12}$ por conseguinte, a melhor forma de eliminar um possível distúrbio na economia seria resultado da total confiança dos agentes econômicos em relação ao comportamento médio dos preços no futuro. ${ }^{13}$ Nesse sentido, a política monetária poderia desempenhar um importante papel para a estabilidade da economia. Em situações como a de um orçamento federal explosivo, que poderia implicar ocorrência de déficits capazes de gerar inflação, a política monetária poderia reduzir o custo social por meio de uma taxa mais lenta de crescimento monetário. A idéia por trás deste movimento, é que se adotando essa medida, a propagação inflacionária seria bloqueada por uma elevação temporária das taxas de juros.

Em relação ao nível de preços, observa-se que as ligações políticas, embora existentes, entre a ação da autoridade monetária e a variação do nível de preços, se manifestam de forma indireta. $\mathrm{Na}$ visão friedmaniana, a autoridade monetária deve evitar oscilações bruscas no comportamento da política monetária. Isto se explica pelo fato de que as autoridades monetárias tomam suas decisões em função da conjuntura atual, entretanto, suas ações somente afetarão a economia daqui a algum tempo. Sendo assim, há a propensão da autoridade monetária lançar mão

${ }^{11}$ É importante ressaltar que a "(...) discretion is the special case of a rule or contract in which none of today's provisions restrict a person's future actions.” (Barro, 1986: 23)

${ }^{12}$ As expectativas são formadas a partir da experiência passada, recebendo maior ênfase os períodos mais recentes.

13 Antigamente, o padrão-ouro servia como uma espécie de âncora que garantia a estabilidade monetária futura. Como esse padrão não é mais o comum sendo predominante o de caráter fiduciário, Friedman (1968) propôs que a autoridade monetária funcionasse como um novo indicador determinando as taxas de câmbio por meio do volume de moeda em circulação em resposta ao fluxo do balanço de pagamentos. 
de flutuações inesperadas sobre a economia para alcançar os objetivos pretendidos pelo governo. Em outras palavras, pode-se dizer que o responsável pela política está sujeito ao chamado viés inflacionário.

De acordo com esta interpretação, é importante que sejam criados mecanismos que evitem tais flutuações. O mecanismo sugerido por Friedman, diz respeito à adoção de uma regra, onde a política monetária deve ser limitada por uma taxa de expansão monetária predeterminada. Dessa forma, seria produzida, em média, uma inflação ou deflação moderada. Nas palavras de Friedman (1968: 17):

“(With)... setting itself a steady course and keeping to it, the monetary authority could make a major contribution to promoting economic stability. By making that course one of steady but moderate growth in the quantity of money, it would make a major contribution to avoidance of either inflation or deflation of prices.”

A antiga literatura sobre regras versus discricionariedade centrava-se nas intenções e na capacidade do responsável pela política. Argumentos favoráveis ao uso de regras tinham por base o conhecimento imperfeito sobre a economia e a tendência das autoridades políticas em conduzir a política econômica para fins inadequados do ponto de vista do bem-estar social. Esta perspectiva sobre regras ou discrição foi alterada pelo artigo de Kydland \& Prescott (1977), que passaram a utilizar as regras como um compromisso (commitment) para a autoridade política. ${ }^{14}$

\subsection{O exemplo fundamental para a teoria}

Para que se possa compreender como se manifesta o problema de inconsistência temporal, recupera-se a seguir o exemplo tradicional da literatura sobre regras versus discricionariedade a partir da análise de Kydland \& Prescott (1977) e Barro \& Gordon (1983). Estes modelos tem por base a estrutura presente na curva de Phillips e possuem duas hipóteses fundamentais:

i) a política monetária afeta o nível geral de preços e a autoridade monetária possui controle perfeito sobre o nível de preços à cada período; $\mathrm{e}$

ii) aumentos não esperados no nível de preços expandem a atividade econômi ca real.

A estrutura básica do exemplo pode ser sumariada da seguinte forma: admita que a autoridade monetária pretende implementar uma regra política ótima ou um plano para a política futura em algum instante no tempo t. Portanto, o responsável pela política maximiza alguma função-objetivo sujeita às necessidades do setor privado. Por conseqüência, uma importante consideração a ser feita é como a política esperada em $t+\Delta t$ afeta as decisões econômicas dos agentes privados no inter-

\footnotetext{
${ }^{14}$ Kydland \& Prescott discutem várias áreas de políticas públicas onde o compromisso é importante. Um exemplo é o caso de patentes que incentivam as invenções, mas também restringem a oferta de bens ex-post.
} 
valo de tempo entre t e $\mathrm{t}+\Delta \mathrm{t}$. Ademais, é necessário verificar se do ponto de vista da autoridade monetária o plano ótimo ex-ante continua sendo ótimo ex-post dada as restrições que se observa em $t+\Delta t$. Para a seqüência do exemplo, é assumido que o plano ótimo ex-ante torna-se inadequado para $t+\Delta t$. A justificativa para esta situação advém do fato de que as decisões do setor privado foram tomadas antes de $t+\Delta t$, o que por sua vez permite a escolha de uma nova estratégia de política pela autoridade monetária. Dessa forma, o planejamento inicial tornou-se inconsistente no tempo.

Deve-se ressaltar que no caso da autoridade política estar condicionada por um mecanismo de incentivo (commitment technology) que a impede de se desviar dos objetivos previamente determinados, o problema de inconsistência temporal ou o desenho institucional não importa para a análise. Entretanto, na maioria dos casos, as autoridades políticas adotam um comportamento discricionário que impede uma previsão acurada para as decisões dos agentes privados mesmo sob expectativas racionais. Além disso, o uso de mecanismos de incentivo para a autoridade política na consecução de determinados objetivos, não representa a escolha ótima para o responsável pela política (na verdade, representa um equilíbrio de second best), o que por conseguinte, motiva a autoridade monetária a buscar o equilíbrio de first best; todavia, o resultado final implica um equilíbrio de third best.

$\mathrm{O}$ que foi dito acima pode ser observado com maiores detalhes da seguinte forma (importante para que se observe a fundamentação teórica). É assumido que a autoridade monetária tem preferência por uma menor taxa de desemprego mesmo ao custo de uma inflação mais elevada, e que os trabalhadores são representados por um sindicato preocupado em garantir a estabilidade do salário real. Ademais, são admitidas duas opções para a política monetária:

i) uma baixa expansão monetária (representada por $\Delta \mathrm{M}_{\mathrm{B}}$ ); ou,

ii) uma alta expansão monetária $\left(\Delta \mathrm{M}_{\mathrm{A}}\right)$.

Com base na Teoria Quantitativa da Moeda, é assumido que o aumento da oferta monetária determina a taxa de inflação e que a taxa de crescimento monetário e a taxa de inflação são iguais. Portanto, quando $\mathrm{M}_{\mathrm{B}}$ é escolhido isto implica uma inflação baixa $\left(\Delta \mathrm{P}_{\mathrm{B}}\right)$ e quando $\mathrm{M}_{\mathrm{A}}$ é escolhido obtém-se uma inflação alta $\left(\Delta \mathrm{P}_{\mathrm{A}}\right)$. Por outro lado, o sindicato negocia os salários nominais com base nas expectativas sobre a política monetária - e portanto da inflação - que vai ser escolhida. Isto é, o sindicato busca um aumento dos salários que corresponda à inflação esperada. Ou seja, se o sindicato espera $\Delta \mathrm{M}_{\mathrm{B}}$ haverá negociação para que seja obtido um baixo aumento salarial $\left(\Delta \mathrm{W}_{\mathrm{B}}\right)$, entretanto se for esperado $\left(\Delta \mathrm{M}_{\mathrm{A}}\right)$ o sindicato tentará obter um alto aumento salarial $\left(\Delta \mathrm{W}_{\mathrm{A}}\right)$.

A partir do que foi dito, há quatro resultados possíveis para inflação e desemprego (tabela 1). Quando a inflação esperada for igual ao salário esperado $(\triangle \mathrm{P}=\mathrm{DW})$, isto é, não há erro de interpretação dos agentes quanto à política monetária a ser adotada, vigora a taxa natural de desemprego $\left(\Delta \mathrm{U}_{\mathrm{N}}\right)$. Quando a inflação for maior que a variação no salário nominal $(\Delta \mathrm{P}>\Delta \mathrm{W})$, a taxa de desemprego $(\Delta \mathrm{U})$ situa-se abaixo da taxa natural de desemprego $\left(\Delta \mathrm{U}_{\mathrm{N}}\right)$ devido à ilusão monetária dos agentes. Por último, no caso de um aumento dos preços inferior ao dos salários $(\Delta \mathrm{P}<$ 
$\Delta \mathrm{W})$ a taxa de desemprego é maior do que aquela definida pela taxa natural de desemprego.

Tabela 1

\begin{tabular}{cccc}
\hline Equil. & $\Delta \mathrm{M}$ e $\Delta \mathrm{W}$ & inflação $(\Delta \mathrm{P})$ & desemprego $(\Delta \mathrm{U})$ \\
\hline 1 & $\Delta \mathrm{MB}_{\mathrm{B}}$ e $\Delta \mathrm{W}_{\mathrm{B}}$ & $\Delta \mathrm{PB}_{\mathrm{B}}$ & $\Delta \mathrm{U}=\Delta \mathrm{U}_{\mathrm{N}}$ \\
2 & $\Delta \mathrm{MB}_{\mathrm{B}}$ e $\Delta \mathrm{W}_{\mathrm{A}}$ & $\Delta \mathrm{P}_{\mathrm{B}}$ & $\Delta \mathrm{U}>\Delta \mathrm{U}_{\mathrm{N}}$ \\
3 & $\Delta \mathrm{M}_{\mathrm{A}}$ e $\Delta \mathrm{W}_{\mathrm{A}}$ & $\Delta \mathrm{P}_{\mathrm{A}}$ & $\Delta \mathrm{U}=\Delta \mathrm{U}_{\mathrm{N}}$ \\
4 & $\Delta \mathrm{M}_{\mathrm{A}}$ e $\Delta \mathrm{W}_{\mathrm{B}}$ & $\Delta \mathrm{P}_{\mathrm{A}}$ & $\Delta \mathrm{U}<\Delta \mathrm{U}_{\mathrm{N}}$ \\
\hline
\end{tabular}

A decisão da autoridade monetária para a escolha da política a ser implementada é resultado da seguinte observação. Se o sindicato escolheu $\Delta \mathrm{W}_{\mathrm{B}}$, a autoridade monetária escolherá $\Delta \mathrm{P}_{\mathrm{A}}$, pois dessa forma é possível obter um desemprego abaixo daquele que corresponde ao da taxa natural. Da mesma forma, se o sindicato optar por $\Delta \mathrm{W}_{\mathrm{A}}$, a autoridade escolherá $\Delta \mathrm{M}_{\mathrm{A}}$ para que o desemprego não fique acima da taxa natural de desemprego. Portanto, independente da escolha do sindicato, a autoridade monetária escolherá a política monetária que implica maior inflação.

Esta constatação não representa informação privilegiada para a autoridade monetária, por conseqüência, o sindicato escolherá a opção que corresponde a um alto aumento salarial $\left(\Delta \mathrm{W}_{\mathrm{A}}\right)$ na tentativa de proteger o salário real dos trabalhadores. Assim, observa-se que o equilíbrio a ser obtido corresponde àquele onde se observa uma alta inflação e vigora a taxa natural de desemprego (equilíbrio 3 third best $-\Delta \mathrm{M}_{\mathrm{A}}$ e $\Delta \mathrm{W}_{\mathrm{A}}$, vide tabela 1).

Não obstante o resultado encontrado, observa-se que a mesma taxa de desemprego, o mesmo salário real e uma inflação mais baixa poderia ser obtida se o resultado obtido correspondesse ao equilíbrio 1 (second best $-\Delta \mathrm{M}_{\mathrm{B}}$ e $\Delta \mathrm{W}_{\mathrm{B}}$ ). Como forma de tornar este equilíbrio factível admita que a autoridade monetária anuncia no início do período que seguirá uma política monetária que implica baixa inflação. Isto é, a autoridade monetária promete ao sindicato que $\Delta \mathrm{M}_{\mathrm{B}}$ será escolhido. Suponha ainda que, com base nessa promessa, o sindicato escolha $\Delta \mathrm{W}_{\mathrm{B}}$. Eis o ponto crucial, após definido o aumento salarial pelo sindicato, a autoridade monetária tem um incentivo em não cumprir a política anunciada, escolhendo $\Delta \mathrm{M}_{\mathrm{A}}$ ao invés de $\Delta \mathrm{M}_{\mathrm{B}}$, pois dessa forma é possível obter um desemprego menor (equilíbrio 4 first best $-\Delta \mathrm{M}_{\mathrm{A}}$ e $\Delta \mathrm{W}_{\mathrm{B}}$ ).

Como o sindicato sabe do incentivo que a autoridade monetária tem para inflacionar a economia após a decisão por um baixo aumento salarial, o anúncio de uma política de baixa expansão monetária tende a não ser crível, o que por conseguinte, implica que o equilíbrio de baixa inflação (equilíbrio 1 - second best) tende a ser inatingível. Assim, o resultado mais provável é que a economia encontre um equilíbrio final com alta inflação (equilíbrio 3 - third best $-\Delta \mathrm{M}_{\mathrm{A}}$ e $\Delta \mathrm{W}_{\mathrm{A}}$ ). De forma sintética, pode-se dizer que a escolha de uma política de alta expansão monetária representa a estratégia da autoridade monetária consistente no tempo, pois 
é a que seria seguida por uma autoridade racional livre. Por outro lado, a estratégia de baixa expansão monetária representa a política ideal do ponto de vista do anúncio inicial, mas é inconsistente no tempo, pois há a tentação da autoridade monetária trapacear.

Diante do exemplo apresentado, observa-se que a análise sobre credibilidade está relacionada a duas questões básicas:

i) questão positiva: como o setor privado responde às escolhas feitas pelo responsável pela política?

ii) questão normativa: a escolha política realizada implica o melhor resultado em termos de bem-estar social?

A questão positiva remete a uma análise, cujo resultado é endógeno ao processo de formação política. Por conseguinte, o foco da análise normativa também muda: se o equilíbrio político depende das instituições no processo de elaboração das políticas, algumas instituições devem ser mais indicadas para o alcance do bem-estar social do que outras (Persson \& Tabellini, 1994). Sob esta perspectiva ganhou destaque a proposta de independência do $\mathrm{BC}$ para a condução da política monetária.

\section{AS GERAÇÕES DOS MODELOS DE IBC}

A premissa básica da proposição de IBC é a busca da estabilidade de preços. Existe a crença de que BCIs são mais confiáveis para utilizar políticas de desinflação, e que, portanto, tais instituições recebem um bônus correspondente à credibilidade, o que reduz o custo de manter a inflação baixa.

Em linhas gerais, dois argumentos básicos são utilizados para justificar a adoção de um BCI:

(i) o viés inflacionário de políticas discricionárias (neste caso, a análise deriva do debate "regras versus discricionariedade") — a análise padrão sobre o comportamento discricionário da política monetária indica que há um incentivo para as autoridades monetárias reduzir o desemprego por meio de políticas inflacionárias. O problema consiste no fato de que no longo termo o efeito de uma expansão monetária sobre o emprego desaparece, enquanto que o aumento da inflação perdura. Em contrapartida, se forem adotadas regras, o viés inflacionário fica impedido de se manifestar, o que implica ser um resultado preferível ao comportamento discricionário. Sob esta perspectiva, um importante mecanismo institucional capaz de evitar a ação discricionária do governo seria o uso de agências independentes.

(ii) uso político da política monetária (derivado da análise sobre dominânciafiscal e ciclo político eleitoral) - admitindo-se que há distinção entre a autoridade monetária e a fiscal, sendo a última predominante (Sargent \& Wallace, 1981); se o público não estiver disposto a absorver um déficit adicional, as autoridades monetárias serão forçadas a financiar o governo via emissão de moeda. Por outro lado, assumindo-se a autoridade monetária como dominante, as autoridades fiscais serão forçadas a reduzir o déficit (ou não reconhecer parte da dívida). Quanto ao ciclo 
político eleitoral, observa-se que governos em períodos próximos às eleições tendem a preferir maior nível de emprego à menor taxa de inflação, pois acreditam que dessa forma conseguirão se reeleger ou favorecer a eleição dos candidatos de seu partido político. Assim, há uma tendência dos governos de pressionarem os responsáveis pela política monetária para que implementem políticas que visem o aumento do produto. Isso implica dizer que, se houver na economia um BC subserviente, existe uma propensão a expandir a oferta monetária com o objetivo de reduzir o nível de desemprego. Em suma, períodos de mudança de governo tendem a provocar maior variabilidade da inflação, sobretudo se as autoridades monetárias forem dominadas por eleições políticas.

A partir dos argumentos supracitados pode-se identificar três gerações para a análise de independência do BC. A primeira geração dos modelos de IBC teve origem com Rogoff (1985). Na interpretação dada por Rogoff, a função de perda social considera os desvios tanto da inflação quanto do produto em relação ao nível considerado ótimo. Esta perda pode ser reduzida quando se adota um modelo de vários períodos no qual o BC procura desenvolver uma reputação. Ainda de acordo com Rogoff, o mesmo resultado pode ser obtido por meio da delegação da política monetária a uma pessoa ou instituição que tenha mais atenção a função de bem-estar social, ou seja, um central banker com características conservadoras. ${ }^{15}$

Deve-se ressaltar que, na estrutura presente nos modelos de primeira geração, o conceito de independência é resultado de outros dois: independência operacional (instrument independence) e independência de meta (goal independence). No primeiro caso, há a necessidade de que o BC tenha à sua disposição os instrumentos necessários para que possa alcançar seus objetivos sem depender de nenhuma outra autoridade política. Por outro lado, a independência de meta refere-se à liberdade que o BC possui para definir qual o objetivo a ser alcançado (Fischer, 1995).

O principal problema apresentado pelos modelos de primeira geração é que a estrutura elaborada não é capaz de alcançar o ótimo de bem-estar, dado que a redução do viés inflacionário é obtido às custas de menor estabilização do produto.

Além disso,

"One major limitation of Rogoff's analysis, (...) is that it is done in the context of a simple static model, where the private sector of the economy is described by a simple 'surprise' supply function" (Lockwood, Miller $\&$ Zhang, 1998: 328).

Nos modelos de segunda geração não é mais discutido qual deve ser o papel do BC. A idéia de que o BC tem como meta natural a busca da estabilidade de preços faz parte das premissas desses modelos, ou seja, o conceito de independência,

\footnotetext{
${ }^{15}$ Uma outra observação a ser feita refere-se à análise elaborada por Lohmann (1992) explorando o tradeoff comprometimento e flexibilidade (commitment and flexibility). Os resultados obtidos indicam que, na presença de choques de oferta e de conflito entre a autoridade monetária e a fiscal, seria adequada a escolha de um central banker conservador para BCs parcialmente independentes.
} 
neste caso, está associado apenas à independência operacional. Sob esta análise, a principal questão a ser respondida passa a ser qual o melhor desenho institucional para evitar o viés inflacionário, uma vez que o conservadorismo do central banker não consiste em uma solução factível para o problema.

Dentre os diversos trabalhos que representam essa geração, ${ }^{16} \mathrm{a}$ análise feita por Walsh (1995) é de forma indubitável a que representou uma evolução em relação aos modelos anteriores. A interpretação dada por Walsh (1995) incorpora o papel dos contratos ótimos entre o $\mathrm{BC}$ e o governo tendo como base a teoria do agenteprincipal. ${ }^{17} \mathrm{O}$ problema do viés inflacionário é resolvido pela estruturação de um contrato que impõe custos ao BC quando a inflação se desvia do nível ótimo. Em outras palavras, sob esta concepção o principal (governo) assina um contrato de incentivo com um agente (BC), segundo o qual o agente fica sujeito ao conjunto de penalidades ex-post, que será determinado pelo desvio da inflação à meta preestabelecida. A vantagem desta estrutura advém do fato de que o melhor resultado para a sociedade é obtido, independente de o governo e o BC partilharem da mesma função-objetivo e do mesmo conjunto de informações. O ponto central desta corrente é que em sociedades democráticas o $\mathrm{BC}$ deve prestar contas ao público. ${ }^{18}$

Os modelos de segunda geração têm como característica o fato de que o governo implementa um contrato (incentive scheme) com o $\mathrm{BC}$ de forma que o retorno financeiro deste último seja inversamente relacionado com a taxa de inflação. O problema com esta estrutura é que ela não é suficiente para superar a inconsistência dinâmica.

“(...) the government has exactly the same incentive not to do so as is identified by Kydland-Prescott (1977) and Barro-Gordon (1983) analysis. Indeed, if the absence of any precommitment technology is actually a problem, then it must apply to the consolidated central-bank-government entity just as it would to an entirely independent central bank. If the technology does not exist, then it does nor exist. Nor is this problem overcome by saying that the objective function must be specified at the 'constitutional stage' of the political process. Again the problem is that constitutions need to be enforced." (McCallum, 1995: 210)

\footnotetext{
${ }^{16}$ Ver Lohmann (1992), Persson \& Tabellini (1994) e Walsh (1995).

${ }^{17}$ Conforme destacado por Walsh (1995, p. 150, grifo meu) "[The literature on independence of central banks] (...) has viewed the incentive problem as one involving many principals (the individuals in the economy) and one agent (the central banker). In this framework, the principals may select the agent, but they are unable to specify the objective function of the agent. However, in no country is the institutional framework such that the actual agents in charge of monetary policy are directly chosen by the individual citizens. Instead, citizens in democracies choose a government, and the central-bank head is chosen by government. Thus, monetary policy involves a multilevel principal-agent problem."

$18 \mathrm{O}$ exemplo mais notório que tem inspirado a adoção deste comportamento em diversos países é o caso do Banco da Nova Zelândia.
} 
É importante salientar que a identificação desta impropriedade, não deve ser interpretada como uma recusa da utilidade de arranjos institucionais tais como o adotado pela Nova Zelândia, em que o BC é submetido a um contrato cuja performance é avaliada em termos da consecução da meta inflacionária estabelecida. $\mathrm{O}$ elemento que merece destaque é que o cumprimento desses contratos, em períodos em que o desemprego encontra-se acima da média, inibe a tentativa do governo de fazer uso do imposto inflacionário.

Contudo, se a taxa de desemprego permanecer elevada por um longo período, é provável que as pressões da sociedade sobre o governo, e por conseguinte sobre o BC, impliquem um esforço maior da autoridade monetária na busca da meta anunciada ou então na revogação do contrato. Portanto, sob essa estrutura, em períodos de alto nível de desemprego, há o aumento do risco da perda da credibilidade alcançada pela autoridade monetária e, por conseguinte, a possibilidade de maior custo para a estabilidade de preços no futuro.

Além disso, “(...) planejadores centrais não existem na prática. Daí ter-se de confiar que o governo imponha o melhor plano de incentivo ao BC ex-post" (Cukierman, 1996: 406). Entretanto, conforme salientam McCallum (1995) e Walsh (1995), se não houver um conjunto ótimo de penalidades previamente à realização dos diversos tipos de contratos, o contrato ótimo do BC não terá credibilidade.

Um outro elemento que permeia os modelos de segunda geração diz respeito à questão democrática. Esta questão foi merecedora de atenção, pois a delegação de autoridade para um BCI, sugerida pelos modelos de primeira geração, foi criticada por não adotar uma estrutura democrática, uma vez que os responsáveis pelo BC não são escolhidos via votação. Por outro lado, os defensores dos modelos de primeira geração, defendem a idéia da delegação mantendo o argumento de que caso os dirigentes fossem escolhidos via votação, este procedimento viria a contribuir no aumento do viés inflacionário. ${ }^{19}$ Daí a idéia presente nos modelos de segunda geração de que o BC deve prestar contas ao público.

Autores de cunho keynesiano têm argumentado que sob o ponto de vista democrático a IBC deve ser limitada, de forma que os responsáveis pela política monetária não possam desviar-se daquilo que as pessoas esperam que venha ocorrer quando da eleição dos seus representantes. Conforme é destacado por Bain, Arestis e Howells 1996: 234-235)

"It is accepted that an independent central bank is given an objective (or objectives) in relation to which its performance can be judged. This, together with requirements that the central bank governor report regularly to the government or the parliament or one of its committees, is held to provide the necessary democratic accountability. Independent central banker are then reduced to the level objective technicians who expertly

\footnotetext{
${ }^{19}$ Para uma leitura sobre a questão envolvendo o aspecto democrático, ver Levy (1995-96) e Rymes (199596).
} 
use the instruments available to them to meet targets which are set taking into account 'the long-term interests of the economy as a whole'."

Quando é designado ao BC a busca da estabilidade de preços e há liberdade do uso de instrumentos na busca desse objetivo é entendido que foi concedido maior autonomia para o BC. Conforme salienta Rymes (1995-96), para evitar o perigo de maior autonomia transformar-se em autocracia, nos quais alguns elementos democráticos são violados, a autonomia deve ser balanceada pela explicabilidade (accountability) das ações do BC. O ponto crucial a ser destacado, de acordo com o mesmo autor, é que o BC não deve ser responsável pela estabilidade de preços, que é responsabilidade do governo, mas pela produção de tal estabilidade. Em outras palavras, em uma estrutura do tipo agente-principal, o governo (principal) determina o que a estabilidade de preços significa, cabendo ao BC (agente) a busca da meta pretendida pelo governo.

$\mathrm{Na}$ tentativa de eliminar os problemas mencionados, os modelos de terceira geração se caracterizam pela combinação dos argumentos presentes nos modelos anteriores. Svensson (1997) desenvolveu um modelo em que utiliza a delegação de autonomia a um $\mathrm{BC}$ com característica conservadora associado ao nível de inflação desejado. Ou seja, o autor incorpora no mesmo modelo os dois principais elementos teóricos presentes nos modelos pró-independência.

Svensson (1997) argumenta que um contrato linear, tal como é proposto em Walsh (1995), é uma forma muito elegante de remover o viés inflacionário. Entretanto, existem dificuldades práticas e políticas para implementá-lo. A dificuldade prática resulta do fato de que o custo linear representa um custo monetário. Enquanto que,

"A political difficult is that the contracts stipulates higher monetary rewards to the Governor or board when inflations is low, which may be provocative to the public if correlated with higher unemployment" (Svensson, 1997: 105).

No modelo de Svensson (1997), um regime de meta inflacionária é interpretado como um arranjo do agente e o principal, em que a sociedade (o principal) delega a política monetária ao $\mathrm{BC}$ (o agente). Também é assumido que o $\mathrm{BC}$ tem controle perfeito sobre a inflação. O resultado encontrado indica que pode ser obtido um equilíbrio que corresponda a uma regra ótima sob compromisso.

Apesar do resultado encontrado por Svensson mostrar-se atraente, McCallum (1995) revelou uma contradição na literatura sobre IBC que não deve ser desprezada. Se o principal (a sociedade) tem a mesma função de bem-estar que o BC (o agente), não há motivo para uma relação ótima do tipo agente-principal via contratos ou arranjos de metas.

Além disso, contratos e metas podem, quando aplicados em conjunto, remover o viés inflacionário da condução da política monetária. De acordo com os argumentos de Muscatelli (1998), a solução de não delegação para um BC deve ser 
preferível quando há incerteza quanto às suas preferências. Ademais, deve ser levado em consideração o nível de consenso que existe na sociedade sobre os custos e benefícios da inflação e a estabilização do produto. Ainda sob a interpretação de Muscatelli, a presença de incerteza sobre as preferências do BC estimula o tradeoff entre a política de estabilização do produto e o controle inflacionário. Destarte, de acordo com o autor, a possível solução para atenuar o viés inflacionário decorrente da incerteza sobre as preferências do $\mathrm{BC}$ seria a consideração de independência de meta para o $\mathrm{BC}^{20}$ ou a aplicação de contratos e metas conjuntamente. Nesse sentido, a interpretação de Svensson (1997) é passível de crítica:

"The notion that society will set an inflation target to a CB and that this target is never met does not seem to correspond to the observation that, where explicit targets have been adopted in recent years (Canada, New Zealand, the United Kingdom) actual inflation has tended to converge to the target over time. In addition, negative inflation targets are not observed in practice. The (...) result on joint targets and contracts is because it suggest that, where CBs perceive an additional (implicit or explicit) 'contractual' penalty on inflation, the Svensson conclusion could be reversed." (Muscatelli, 1998: 535)

\section{UMA REGRA PARA A DETERMINAÇÃO DA TAXA DE JUROS}

Ainda que a maior parte dos economistas considere a incapacidade de o BC afetar variáveis reais (crescimento do produto e emprego) no longo prazo, isto não implica que a autoridade monetária não deva estabilizar o emprego no curto prazo, ou que os efeitos provenientes da estabilização de preços sejam ignorados. O motivo para esta conduta tem por base a observação de que o BC afeta a inflação por meio de sua influência sobre o emprego e a atividade real. Portanto, é esperado que na tentativa de estabilizar a inflação o emprego apresente maior instabilidade como forma de compensar os efeitos oriundos da inflação.

Uma outra observação a ser feita, refere-se à afirmação de que a busca da estabilidade de preços como objetivo prioritário evita a perda da credibilidade pela autoridade monetária, decorrente da tentativa de alcançar diversos objetivos. ${ }^{21}$ Todavia, na prática, é comum a interferência do BC sobre o produto e o emprego, o que revela que a ação da autoridade monetária não se restringe a uma única variá-

\footnotetext{
${ }^{20}$ Quando considerado na análise a possibilidade de informação privilegiada do BC sobre os choques de oferta, o modelo de independência de meta apresenta algumas insuficiências - o BC perde o incentivo em atingir as metas inflacionárias e o problema do viés inflacionário volta a existir.

${ }^{21}$ Reminiscência da análise de Tinbergen (1952).
} 
vel ao longo do tempo. Assim sendo, o problema de inconsistência dinâmica pode emergir devido à manifestação do viés inflacionário.

Portanto, o uso de uma regra crível capaz de remover o viés inflacionário e estabilizar de forma simultânea a inflação e o produto, é de grande relevância para a economia. Nos últimos anos, a partir da proposição de Taylor (1993) para a determinação da taxa de juros, diversos teóricos têm dedicado atenção para avaliar se a aplicação da regra proposta é factível. ${ }^{22}$

A proposta de Taylor estabelece uma regra simples para a determinação da taxa de juros com base em quatro fatores básicos:

"The first factor is the current inflation rate. The second factor is the equilibrium real interest rate. When added together, these two factors provide a benchmark recommendation for the nominal federal funds rate. The third factor is an inflation gap adjustment factor based on the gap between the inflation rate and a given target for inflation. (...) The fourth factor is an output gap adjustment factor based on the gap between real GDP and potential real GDP" (Kozicki, 19990: 6, grifos meus).

Os dois primeiros fatores servem de referência para o caso em que a economia está operando ao nível potencial e revela a hipótese implícita do uso de uma taxa real de juros constante. ${ }^{23} \mathrm{O}$ terceiro e o quarto fator da regra de Taylor representam os dois objetivos da política monetária, onde os parâmetros de ajuste referemse ao trade-off de curto prazo entre a inflação e o produto. O terceiro fator implica uma elevação (redução) da taxa de juros quando a inflação encontra-se acima (abaixo) da meta. De forma análoga, o quarto fator indica que no caso de uma diferença positiva (negativa) entre o PIB real e o PIB potencial deve haver um aumento (declínio) na taxa de juros. Ou seja, de acordo com a proposta de Taylor, a taxa básica de juros é obtida por meio de:

$\mathrm{i}_{\mathrm{t}}={ }_{\mathrm{t}}+\mathrm{r}^{*}+0,5\left({ }_{\mathrm{t}}-\pi^{*}\right)+0,5\left(\mathrm{y}_{\mathrm{t}}\right)$, onde

$\mathrm{i}=$ taxa básica de juros nominais; $\mathrm{r}^{*}=$ taxa real de juros de equilíbrio;

$\pi$ = taxa média da inflação dos últimos quatro trimestres (deflator do PIB);

$\pi^{*}=$ meta da taxa de inflação; e

$\mathrm{y}=$ hiato do produto $[100$.(PIB real - PIB potencial $) \div$ PIB potencial $].{ }^{24}$

\footnotetext{
${ }^{22}$ Conforme destacado por Judd \& Rudebusch (1998: 5-6) “(...) the original Taylor rule fits reasonably well to the actual funds rate during the Greenspan period. The $\mathrm{R}^{2}$ for the period is 87 percent for quarterly levels of the nominal funds rate, and 52 percent for quarterly changes. The arguments in the rule - inflation and the GDP gap — roughly correspond with goals legislated for US monetary policy — stable prices and full employment."

${ }^{23}$ Deve-se ressaltar que a taxa real de juros desempenha papel relevante na formulação da política monetária. Apesar de a taxa de juros nominal ser a variável de ajuste da autoridade monetária, é a taxa real de juros que afeta o lado real da economia.

24 Taylor (1993) utilizou uma tendência linear do logaritmo do PIB real com base no período compreendido entre 1984 (primeiro trimestre) e 1992 (terceiro trimestre) como proxy para o produto
} 
Portanto, ao contrário da regra rígida proposta por Friedman (1968) - expansão da oferta monetária a uma taxa constante - a regra proposta por Taylor (1993) corresponde a uma regra de acompanhamento que permite o uso da política monetária quando há desvios em relação às metas de inflação e de produto da economia.

\section{CONSIDERAÇÕES FINAIS}

O estudo realizado permite observar de que forma a maior parte da literatura sobre a credibilidade da política monetária explica a necessidade de concentrar o objetivo da autoridade monetária na busca pela estabilidade de preços. De forma muito simplificada, pode-se esboçar parte do desenvolvimento da análise derivada do debate "regras versus discricionariedade" da seguinte forma (esquema 1):

\section{Esquema 1}

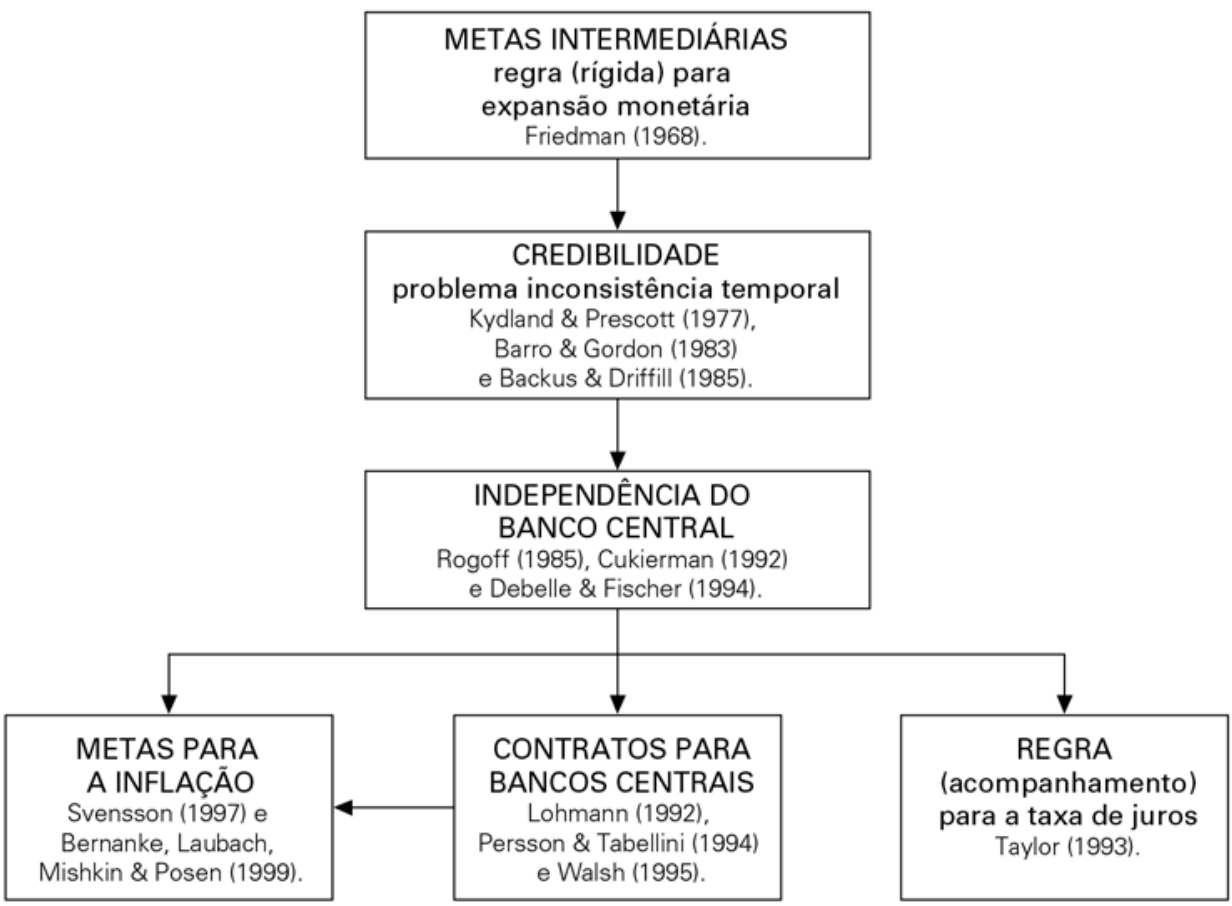

potencial. Uma importante observação a ser feita diz respeito ao fato de que a regra proposta por Taylor para a determinação da taxa básica de juros não é resultado de estimativas econométricas. Foi assumido que o peso dado pelo Federal Reserve aos desvios da inflação e produto são ambas equivalentes a 0,5; e que a taxa real de juros e a inflação são ambas iguais a $2 \%$. Ou seja, a taxa básica de juros reais será igual a $2 \%$ quando a inflação for de $2 \%$ e não houver divergência entre o PIB real e o PIB potencial. Também é observado que para cada ponto percentual de inflação ou do PIB acima (abaixo) de 2\%, a taxa básica de juros aumenta (diminui) $0,5 \%$. 
É importante observar que apesar da teoria que analisa o problema de credibilidade da política monetária apresentar uma consistência interna apreciável, devese chamar atenção para o fato de que a base que sustenta a teoria não é sólida. Para que o exemplo criado por Kydland \& Prescott (1977) e posteriormente desenvolvido por Barro \& Gordon (1983) faça sentido, é assumido que a existência do viés inflacionário derive do fato de o $\mathrm{BC}$ pactuar com o governo na busca de uma taxa de desemprego abaixo da natural, e que para alcançar este objetivo faz uso do imposto inflacionário. Ou seja, há muitas hipóteses ad hoc para a validade da teoria, o que indica ser mais adequado entender grande parte da literatura sobre credibilidade da política monetária como um caso particular, e por conseguinte deve-se ponderar de forma criteriosa a sua aplicabilidade ao mundo real.

Além disso, a teoria que advém do problema do viés inflacionário pressupõe o mau gerenciamento da política monetária. Na tentativa de solucionar esse problema foram desenvolvidas duas estruturas básicas que não foram capazes de apresentar uma solução ótima: o conservadorismo do central banker e a aplicação de contratos ótimos sob a teoria do agente-principal. No período recente, a literatura tem apontado o uso de metas inflacionárias como um importante mecanismo para assegurar a independência operacional do BC. ${ }^{25}$ Não obstante, é importante que não seja desprezada a possibilidade da política monetária causar efeitos reais na economia, nesse sentido, o fato da regra de Taylor incorporar o desvio do produto em relação ao produto potencial representa de forma indubitável um avanço para economistas que acreditam no preceito da neutralidade da moeda.

Deve-se lembrar que do ponto de vista teórico não há consenso entre os economistas de que a única variável que, a longo prazo, pode ser afetada pela política monetária é a taxa de inflação.

"Para darmos apenas alguns exemplos, Robert Eisner, ex-presidente da American Economics Association e renomado especialista em macroeconomia, nunca aceitou a concepção de Friedman-Lucas. Tampouco ela foi aceita por James Tobin, Paul Samuelson, Robert Solow ou pelo finado William Vickrey - todos eles ganhadores do prêmio Nobel. O mesmo se deu com Ray Fair, de Yale; James Medoff, de Havard; e William Dickens, da Brookings Institution. [Além disso], Alan Greenspan, Alice Rivlin e os outros dirigentes do Federal Reserve... [têm] demonstrado quão espúria é a doutrina da taxa natural ao provar que pleno emprego, crescimento equilibrado e uma estabilidade de preços razoável não são mutualmente exclusivos" (Galbraith, 1999: 24, 25).

É diante desse cenário que não deve ser desprezada a possibilidade de coordenação de políticas macroeconômicas como uma alternativa factível para estruturas

\footnotetext{
${ }^{25}$ Para uma leitura sobre o regime de metas inflacionárias e resultados preliminares de sua utilização no Brasil, ver Mendonça (2001).
} 
teóricas que recomendam a concentração de políticas econômicas em um objetivo. A justificativa para este comportamento é simples. Em sociedades democráticas, o cidadão elege seus representantes na expectativa que sejam alcançados diversos objetivos pelo governo, de forma que possa ser alcançado o melhor resultado possível em termos de bem-estar. Como a taxa de inflação representa apenas um dos elementos que o público leva em consideração quando da escolha de seus candidatos, não é justo que, após eleito, o representante da sociedade dê prioridade a um objetivo que possa prejudicar o alcance dos demais.

$\mathrm{O}$ argumento acima não implica a negação de que a inflação seja um objetivo relevante de política, mas que ele não é o único. Ademais, nenhum economista rejeita o fato de que alterações nas taxas de juros da economia é capaz de afetar a atividade econômica. Assim, deve-se, no mínimo, ter o cuidado de observar se o uso da política monetária na busca do difundido objetivo da estabilidade de preços não está acarretando custos sociais desnecessários (aumento do déficit, desemprego, etc.).

Deve-se ressaltar que a regra de reação apresentada por Taylor (1993) representa um aparato razoável para a proposição de coordenação de políticas. A estrutura proposta pelo autor é compatível com a desenvolvida por Tinbergen (1952) e Mundell (1960). Ademais, é explícito o uso da política monetária para a busca da estabilidade de preços, mas há a preocupação de que seja evitado um movimento recessivo sobre a economia. Conforme reconhecido por Barro (1986: 35-36, grifo meu):

"The choice between the two objectives - stabilising the general price level versus stabilising nominal GNP - corresponds to the weights one attaches to the validity of the two competing viewpoints about macroeconomics. (Surely one of these views must be correct!)"

Desse modo, por que insistir apenas na primeira opção? A coordenação de políticas macroeconômicas tem a vantagem de considerar os dois objetivos. $\mathrm{Na}$ verdade, os dois propósitos são relevantes, não há motivos a priori para que a política monetária concentre-se em apenas um deles.

\section{REFERÊNCIAS BIBLIOGRÁFICAS}

ARGY, V. “A Post-War History of the Rules vs Discretion Debate”. BNL Quarterly Review, 1988.

BACKUS, D. \& DRIFFILL, J. "Inflation and Reputation”. The American Economic Review, June 1985. BAIN, K., ARESTIS, P. \& HOWELLS, P. “Central Banks, Governments and Markets: an Examination of Central Bank Independence and Power”. Economis et Sociétés, Monnaie et production, Série M. P., No 10, 2-3/1996.

BARRO, R. J. "Recents Developments in the Theory of Rules Versus Discretion". The Economic Journal, vol. 96, 1986.

BARRO, R. J. \& GORDON, D. Rules, "Discretion and Reputation in a Model of Monetary Policy". Journal of Monetary Economics, 12 (1983): 101-121, North-Holland.

BERNANKE, B., LAUBACH, T., MISHKIN, F. \& POSEN, A. Inflation Targeting: Lessons from the International Experience. Princeton University Press, Princeton, 1999. 
CLARIDA, R., GALI, J. \& GERTLER, M. “The Science of Monetary Policy: A New Keynesian Perspective”. NBER Working Paper N. 7147, May 1999.

CUKIERMAN, A., \& MELTZER, A. H. "A Theory of Ambiguity, Credibility, and Inflation Under Discretion and Asymmetric Information”. Econometrica, 54(5): 1099-1128, September 1986.

CUKIERMAN, A., WEBB, S., \& NEYAPTI, B. "Measuring the Independence of Central Banks and Its Effects on Policy Outcomes". World Bank Economic Review, 6(3), 1992.

CUKIERMAN, A. Central Bank Strategy, Credibility, and Independence: Theory and Evidence. Cambridge, Mass.: The MIT Press, 1992.

. "A Economia do Banco Central”. Revista Brasileira de Economia, Rio de Janeiro 50 (4), out./ dez. 1996.

DEBELLE, G. \& FISCHER, S. “How Independent Should a Central Bank Be?” In: Fuhrer, J. (ed.) Goals, Guidelines, and Constraints Facing Monetary Policymakers. Federal Reserve Bank of Boston, Boston, 1994.

FISCHER, S. “Central Bank Independence Revisited”. The American Economic Review, May 1995.

FRIEDMAN, M. “Should There Be an Independent Monetary Authority?” In: L. Yeager (ed), In Search of a Monetary Constitution, Cambridge: Harvard University Press, 1962.

"The Role of Monetary Policy". The American Economic Review, March 1968.

GALBRAITH, J.K. "Fixados na Inflação”. Foreign Affairs. Gazeta Mercantil, 1999.

HALDANE, A. G. Targeting Inflation. Bank of England, 1995.

JENSEN, H. "Credibility of Optimal Monetary Delegation". The American Economic Review, December 1997.

JUDD, J.P. \& RUDEBUSCH, G.D. “Taylor's Rule and the Fed: 1970-1997”. Economic Review, Federal Reserve Bank of San Francisco, 1998, n. 3.

KING, M. "How Should Central Banks Reduce Inflation: Conceptual Issues”. Economic Review, Federal Reserve Bank of Kansas City, Fourth Quarter 1996.

KOZICKI, S. “How Useful are Taylor Rules for Monetary Policy?” Economic Review, Federal Reserve Bank of Kansas City, Second Quarter 1999.

KYDLAND, F. E. \& PRESCOTT, E. C. "Rules Rather than Discretion: the Inconsistency of Optimal Plans". Journal of Political Economic, 1977, vol. 85, n. 3.

LEVY, D.A. "Does an Independent Central Bank Violate Democracy?” Journal of Post Keynesian Economics / Winter 1995-96, vol. 18, No.2., p. 189-210.

LOCKWOOD, B., MILLER, M. \& ZHANG, L. "Designing Monetary Policy when Unemployment Persists". Economica, 65, 1998.

LOHMAN, S. "Optimal Commitment in Monetary Policy: Credibility versus Flexibility". The American Economic Review, 82, 1992.

LUCAS Jr., R. "Expectations and the Neutrality of Money”. Journal of Economic Theory, 4, 1972.

. "Some International Evidence on Output-Inflation Tradeoffs". The American Economic Review, June 1973.

McCALlUM, B. T. “Two Fallacies Concerning Central Bank Independence”. The American Economic Review, May 1995.

MENDONÇA, H.F. “Metas de Inflação: Uma Análise Preliminar Para o Caso Brasileiro”. Economia Aplicada, FIPE/FEA-USP, Jan.-Mar., 5 (1), 2001.

MUNDELL, R. "The Monetary Dynamics of International Adjustment under Fixed and Flexible Exchange Rates”, Quarterly Journal of Economics, May 1960.

MUSCATELLI, A. "Optimal inflation Contracts and Inflation Targets with Uncertain Central Bank Preferences: Accountability Through Independence?” The Economic Journal, 108, March 1998.

MUTH, J. F. "Rational Expectations and the Theory of Price Movements". Econometrica, 29(3), July 1961.

PERSSON, T. \& TABELLINI, G. (ed.) Monetary and Fiscal Policy. MIT 1994.

PHILLIPS, A. "Stabilization Policy and the Time Form of Lagged Responses". Economic Journal, 67, 1957. 
ROGOFF, K. “The Optimal Degree of Commitment to an Intermediate Monetary Target”. The Quarterly Journal of Economics, November 1985.

RYMES, T. K. "Autonomous and Accountable". Journal of Post Keynesian Economics, 18(2), Winter 1995-96.

SARGENT, T.J. Rational Expectations, the Real Rate of Interest, and the Natural Rate of Unemployment. Brooking Papers on Economic Activity, 1973.

SARGENT, T.J. \& WALLACE, N. “Some Unpleasant Monetarist Arithmetic”. Federal Reserve Bank of Minneapolis Quarterly Review, Fall 1981: 1-17.

SVENSSON, L. "Optimal Inflation Targets, "Conservative" Central Banks, and Linear Inflation Contracts”. The American Economic Review, 87(1), March 1997.

TAYLOR, J.B. "Discretion Versus Policy Rules in Practice". Carnegie-Rochester Conference Series on Public Policy, N. 39, 1993.

TINBERGEN, J. On the Theory of Economic Policy. Amsterdam: North Holland, 1952.

WALSH, C. “Optimal Contracts for Central Bankers”. The American Economic Review, March 1995, 85 (1). 